\title{
A Coupled Fixed Point Theorem for Three Pairs of $w$-Compatible Mappings in $G$-metric spaces
}

\author{
R. A. Rashwan* and S. M. Saleh* \\ Department of Mathematics, Faculty of Science, Assiut University, Assiut, Egypt
}

Received: 8 Jun. 2013, Revised: 4 Oct. 2013, Accepted: 5 Oct. 2013

Published online: 1 Jan. 2014

\begin{abstract}
In this paper, we establish a common coupled fixed point theorem for three pairs of $w$-compatible maps in $G$-metric space which improve and generalize the results of Ding and Karapnar [5]. Example are presented to support our result.
\end{abstract}

Keywords: $G$-metric space, $w$-compatible mappings, coupled fixed point.

Mathematics Subject Classification: 54H25, 47H10.

\section{Introduction and preliminaries}

The notion of $G$-metric space was introduced by Mustafa and Sims [10], [11] as a generalization of metric spaces. Afterwards Mustafa and Sims [12] proved fixed point theorems for mappings satisfying different contractive conditions in this space.

The notion of coupled fixed point was introduced by Guo and Lakshmikantham [6] in 1987. In a recent paper, Gnana-Bhaskar and Lakshmikantham [3] introduced the concept of mixed monotone property for contractive operators of the form $F: X \times X \rightarrow X$, where $X$ is a partially ordered metric space, and then established some coupled fixed point theorems. They also illustrated these results by proving the existence and uniqueness of the solution for a periodic boundary value problem. Later, Lakshmikantham and Ćirić [8] proved coupled coincidence and coupled common fixed point results for nonlinear mappings satisfying certain contractive conditions in partially ordered complete metric spaces. After that many results appeared on coupled fixed point theory in different contexts (see e.g. [1], [4], [7], [8], [9], [13]-[16]). Recently, Ding and Karapnar [5] extend some recent coupled fixed point theorems in the context of $G$-metric space.

The aim of this paper is to study common coupled fixed point theorem for three pairs of mappings in $G$-metric spaces. Our result generalize the results of Ding and Karapnar [5].
First, we recall the necessary definitions and results which will be useful for the rest of the paper.

Definition 1.[11] Let $X$ be a nonempty set and let $G: X^{3} \rightarrow[0, \infty)$ be a function satisfying:

$\left(G_{1}\right) G(x, y, z)=0$ if $x=y=z$,

$\left(G_{2}\right) 0<G(x, x, y)$, for all $x, y \in X$, with $x \neq y$,

$\left(G_{3}\right) G(x, x, y) \leq G(x, y, z), \forall x, y, z \in X$, with $z \neq y$,

$\left(G_{4}\right) G(x, y, z)=G(x, z, y)=G(y, z, x)=$ $\ldots,($ symmetry in all three variables $)$,

$\left(G_{5}\right) G(x, y, z) \leq G(x, a, a)+G(a, y, z), \forall x, y, z, a \in$ $X,($ rectangle inequality).

Then the function $G$ is called a $G$-metric on $X$, and the pair $(X, G)$ is called a $G$-metric space.

Definition 2.[11] Let $(X, G)$ be a G-metric space, a sequence $\left(x_{n}\right)$ is said to be

(i) G-convergent if for every $\varepsilon>0$, there exists an $x \in X$, and $k \in \mathbf{N}$ such that for all $m, n \geq k, G\left(x, x_{n}, x_{m}\right)<\varepsilon$.

(ii)G-Cauchy if for every $\varepsilon>0$, there exists an $k \in \mathbf{N}$ such that for all $m, n, p \geq k, G\left(x_{m}, x_{n}, x_{p}\right)<\varepsilon$, that is $G\left(x_{m}, x_{n}, x_{p}\right) \rightarrow 0$ as $m, n, p \rightarrow \infty$.

(iii)A space $(X, G)$ is said to be $G$-complete if every $G$ Cauchy sequence in $(X, G)$ is $G$-convergent.

Lemma 1.[11] Let $(X, G)$ be a $G$-metric space. Then the following are equivalent:

(i) $\left(x_{n}\right)$ is convergent to $x$,

(ii) $G\left(x_{n}, x_{n}, x\right) \rightarrow 0$ as $n \rightarrow \infty$,

\footnotetext{
*Corresponding author e-mail: rr_rashwan54@yahoo.com, samirasaleh2007@yahoo.com
} 
(iii) $G\left(x_{n}, x, x\right) \rightarrow 0$ as $n \rightarrow \infty$,

(iv) $G\left(x_{n}, x_{m}, x\right) \rightarrow 0$ as $n, m \rightarrow \infty$,

Lemma 2.[11] Let $(X, G)$ be a G-metric space. Then the following are equivalent:

(i)The sequence $\left(x_{n}\right)$ is G-Cauchy,

(ii)for every $\varepsilon>0$, there exists $k \in \mathbf{N}$ such that $G\left(x_{n}, x_{m}, x_{m}\right)<\varepsilon$ for $m, n \geq k$.

Lemma 3.[11] Let $(X, G)$ be a G-metric space. Then the function $G(x, y, z)$ is jointly continuous in all three of its variables.

Definition 3.[11] A $G$ metric space $X$ is symmetric if $G(x, y, y)=G(y, x, x)$ for all $x, y \in X$.

Proposition 1.[11] Every G-metric space $(X, G)$ will define a metric space $\left(X, d_{G}\right)$ by

$$
d_{G}(x, y)=G(x, y, y)+G(y, x, x), \quad \forall x, y \in X .
$$

Proposition 2.[11] Let $(X, G)$ be a $G$-metric space. Then for any $x, y, z$, and $a \in X$, it follows that

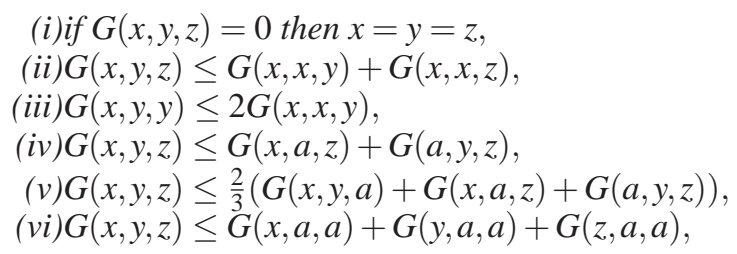

Definition 4.[3] An element $(x, y) \in X \times X$ is called a coupled fixed point of a mapping $F: X \times X \rightarrow X$ if $x=F(x, y)$ and $y=F(y, x)$.

Definition 5.[8] An element $(x, y) \in X \times X$ is called

(i) a coupled coincident point of mappings $F: X \times X \rightarrow X$ and $f: X \rightarrow X$ if $f x=F(x, y)$ and $f y=F(y, x)$,

(ii) a common coupled fixed point of mappings $F: X \times$ $X \rightarrow X$ and $f: X \rightarrow X$ if $x=f x=F(x, y)$ and $y=$ $f y=F(y, x)$.

Definition 6.[2] The mappings $F: X \times X \rightarrow X$ and $f: X \rightarrow X$ are called $w$-compatible if $f(F(x, y))=F(f x, f y)$ whenever $f(x)=F(x, y)$ and $f(y)=F(y, x)$.

\section{Main Results}

Theorem 1.Let $(X, G)$ be a $G$-metric space. Let $S, T, R$ : $X \times X \rightarrow X$ and $f, g, h: X \rightarrow X$ be mappings satisfying the following condition

$$
\begin{aligned}
& G(S(x, y), T(u, v), R(w, z))+G(S(y, x), T(v, u), R(z, w)) \\
& \quad \leq k(G(f x, g u, h w)+G(f y, g v, h z))
\end{aligned}
$$

for all $x, y, u, v, w, z \in X$, where $k \in[0,1)$. Suppose that (i) $S(X \times X) \subseteq g(X), T(X \times X) \subseteq h(X), R(X \times X) \subseteq f(X)$, (ii)either $f(X), g(X)$ or $h(X)$ is a G-complete subspace of $X$.

(iii)the pairs $(f, S),(g, T)$ and $(h, R)$ are $w$-compatible.

Then the pairs $(f, S),(g, T)$ and $(h, R)$ have the same unique common coupled fixed point of the form $(w, w)$ in $X \times X$.

Proof.Let $x_{0}, y_{0} \in X$. Since $S(X \times X) \subseteq g(X), T(X \times X) \subseteq$ $h(X), R(X \times X) \subseteq f(X)$, we can construct sequences $\left\{x_{n}\right\}$, $\left\{y_{n}\right\}\left\{w_{n}\right\}$, and $\left\{z_{n}\right\}$ in $X$ such that

$$
\begin{aligned}
& w_{3 n}=S\left(x_{3 n}, y_{3 n}\right)=g x_{3 n+1}, \quad z_{3 n}=S\left(y_{3 n}, x_{3 n}\right)=g y_{3 n+1}, \\
& w_{3 n+1}=T\left(x_{3 n+1}, y_{3 n+1}\right)=h x_{3 n+2}, \quad z_{3 n+1}=T\left(y_{3 n+1}, x_{3 n+1}\right)=h y_{3 n+2} \text {, and } \\
& w_{3 n+2}=R\left(x_{3 n+2}, y_{3 n+2}\right)=f x_{3 n+3}, \quad z_{3 n+2}=R\left(y_{3 n+2}, x_{3 n+2}\right)=f y_{3 n+3}, \quad \text { for } n \geq 0
\end{aligned}
$$

Let

$M_{3 n}=G\left(w_{3 n}, w_{3 n+1}, w_{3 n+2}\right)+G\left(z_{3 n}, z_{3 n+1}, z_{3 n+2}\right), \quad n \geq 0$.

Then from (1), for all $n>0$, we have

$$
\begin{aligned}
M_{3 n}= & G\left(w_{3 n}, w_{3 n+1}, w_{3 n+2}\right)+G\left(z_{3 n}, z_{3 n+1}, z_{3 n+2}\right) \\
= & G\left(S\left(x_{3 n}, y_{3 n}\right), T\left(x_{3 n+1}, y_{3 n+1}\right), R\left(x_{3 n+2}, y_{3 n+2}\right)\right. \\
& +G\left(S\left(y_{3 n}, x_{3 n}\right), T\left(y_{3 n+1}, x_{3 n+1}\right), R\left(y_{3 n+2}, x_{3 n+2}\right)\right) \\
\leq & k\left(G\left(f x_{3 n}, g x_{3 n+1}, h x_{3 n+2}\right)+G\left(f y_{3 n}, g y_{3 n+1}, h y_{3 n+2}\right)\right) \\
= & k G\left(w_{3 n-1}, w_{3 n}, w_{3 n+1}\right)+G\left(z_{3 n-1}, z_{3 n}, z_{3 n+1}\right) \\
= & k M_{3 n-1} .
\end{aligned}
$$

Similarly, we conclude that

$$
M_{3 n} \leq k M_{3 n-1} \leq k^{2} M_{3 n-2} \leq \cdots \leq k^{3 n} M_{0} .
$$

Therefore

$$
M_{n} \leq k^{n} M_{0} \quad \forall n \geq 0 .
$$

Now, for all $l, m, n$ with $l>m>n$, by using $G_{5}$ and (1) we get

$$
\begin{aligned}
& G\left(w_{n}, w_{m}, w_{l}\right)+G\left(z_{n}, z_{m}, z_{l}\right) \\
& \leq G\left(w_{n}, w_{n+1}, w_{n+1}\right)+G\left(w_{n+1}, w_{n+2}, w_{n+2}\right)+\cdots+G\left(w_{l-1}, w_{l-1}, w_{l}\right) \\
& +G\left(z_{n}, z_{n+1}, z_{n+1}\right)+G\left(z_{n+1}, z_{n+2}, z_{n+2}\right)+\cdots+G\left(z_{l-1}, z_{l-1}, z_{l}\right) \\
& \leq G\left(w_{n}, w_{n+1}, w_{n+2}\right)+G\left(w_{n+1}, w_{n+2}, w_{n+3}\right)+\cdots+G\left(w_{l-2}, w_{l-1}, w_{l}\right) \\
& +G\left(z_{n}, z_{n+1}, z_{n+2}\right)+G\left(z_{n+1}, z_{n+2}, z_{n+3}\right)+\cdots+G\left(z_{l-2}, z_{l-1}, z_{l}\right) \\
& \leq M_{n}+M_{n+1}+\cdots+M_{l-2} \\
& \leq\left(k^{n}+k^{n+1}+\cdots+k^{l-2}\right) M_{0} \\
& \leq \frac{k^{n}}{1-k} M_{0} .
\end{aligned}
$$

Also, if $l=m>n$ and $l>m=n$ we obtain

$G\left(w_{n}, w_{m}, w_{l}\right)+G\left(z_{n}, z_{m}, z_{l}\right) \leq \frac{k^{n}}{1-k} M_{0}$.

Consequently $G\left(w_{n}, w_{m}, w_{l}\right)+G\left(z_{n}, z_{m}, z_{l}\right) \rightarrow 0$ as $n, m, l \rightarrow \infty$. Therefore $\left\{w_{n}\right\}$ and $\left\{z_{n}\right\}$ are $G$-Cauchy sequences. Suppose that $f(X)$ is a $G$-complete subspace of $X$, then there exists points $w, z \in f(X)$ such that $\left\{w_{3 n+2}\right\}$ and $\left\{z_{3 n+2}\right\}$ are convergent to $w$ and $z$, respectively as $n \rightarrow \infty$. Moreover, we can find $x, y \in f(X)$ such that $f x=w$ and $f y=z$. Also, since $\left\{w_{n}\right\}$ and $\left\{z_{n}\right\}$ are $G$-Cauchy sequences then $\lim _{n \rightarrow \infty} w_{n}=w$ and $\lim _{n \rightarrow \infty} z_{n}=z$. 

Since

We claim that $f w=S(w, z)=w$ and $f z=S(z, w)=z$.

$$
\begin{aligned}
& G\left(S(x, y), w_{3 n+1}, w_{3 n+2}\right)+G\left(S(y, x), z_{3 n+1}, z_{3 n+2}\right) \\
& =G\left(S(x, y), T\left(x_{3 n+1}, y_{3 n+1}\right), R\left(x_{3 n+2}, y_{3 n+2}\right)\right) \\
& +G\left(S(y, x), T\left(y_{3 n+1}, x_{3 n+1}\right), R\left(y_{3 n+2}, x_{3 n+2}\right)\right) \\
& \leq k\left(G\left(f x, g x_{3 n+1}, h x_{3 n+2}\right)+G\left(f y, g y_{3 n+1}, h y_{3 n+2}\right)\right) \\
& =k G\left(w, w_{3 n}, w_{3 n+1}\right)+G\left(z, z_{3 n}, z_{3 n+1}\right) .
\end{aligned}
$$

Letting $n \rightarrow \infty$ we have

$$
G(S(x, y), w, w)+G(S(y, x), z, z)=0 .
$$

Hence $S(x, y)=f x=w$ and $S(y, x)=f y=z$. Since $(f, S)$ is $w$-compatible, $S(w, z)=f w$ and $S(z, w)=f z$. From (1) we get

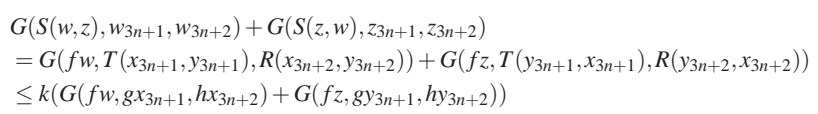

Letting $n \rightarrow \infty$ we have

$$
G(f w, w, w)+G(f z, z, z) \leq k(G(f w, w, w)+G(f z, z, z)) .
$$

Hence $G(f w, w, w)+G(f z, z, z)=0$. Therefore $f w=w$ and $f z=z$. So

$$
f w=S(w, z)=w \quad \text { and } \quad f z=S(z, w)=z .
$$

We prove that $g w=T(w, z)=w$ and $g z=T(z, w)=z$. Since $S(X \times X) \subseteq g(X)$, there exists $u, v \in X$ such that $g u=S(w, z)=w$ and $g v=S(z, w)=z$. From (1) we obtain

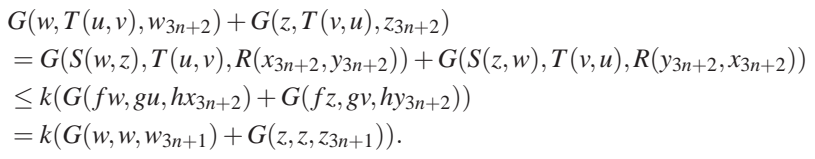

Letting $n \rightarrow \infty$ we have $G(w, T(u, v), w)+G(z, T(v, u), z)=$ 0 , which gives that $T(u, v)=g u=w$ and $T(v, u)=g v=z$. Since $(g, T)$ is $w$-compatible, $T(w, z)=g w$ and $T(z, w)=$ $g z$. From (1) we get

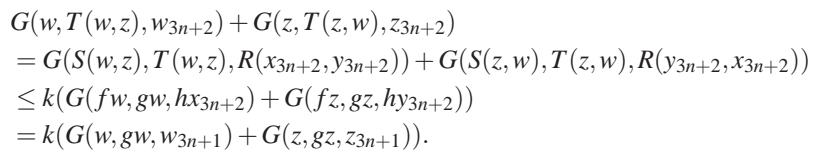

Letting $n \rightarrow \infty$ we have

$$
G(w, g w, w)+G(z, g z, z) \leq k(G(w, g w, w)+G(z, g z, z)) .
$$

Hence $G(w, g w, w)+G(z, g z, z)=0$. Therefore

$$
g w=T(w, z)=w \quad \text { and } \quad g z=T(z, w)=z .
$$

We show that $h w=R(w, z)=w$ and $h z=R(z, w)=z$. Since $T(X \times X) \subseteq h(X)$, there exists $s, t \in X$ such that $h s=$
$T(w, z)=w$ and $h t=T(z, w)=z$. By (1) we obtain

$$
\begin{aligned}
& G(w, w, R(s, t))+G(z, z, R(t, s)) \\
& =G(S(w, z), T(w, z), R(s, t)))+G(S(z, w), T(z, w), R(t, s)) \\
& \leq k(G(f w, g w, h s)+G(f z, g z, h t)) \\
& =k(G(w, w, w)+G(z, z, z))=0 .
\end{aligned}
$$

So we have $G(w, w, R(s, t))=G(z, z, R(t, s))=0$, which gives that $R(s, t)=h s=w$ and $R(t, s)=h t=z$. Since $(h, R)$ is $w$-compatible, $R(w, z)=h w$ and $R(z, w)=h z$. From (1) we conclude that

$$
\begin{aligned}
& G(w, w, h w)+G(z, z, h z) \\
& =G(S(w, z), T(w, z), R(w, z)))+G(S(z, w), T(z, w), R(z, w)) \\
& \leq k(G(f w, g w, h w)+G(f z, g z, h z)) \\
& =k(G(w, w, h w)+G(z, z, h z)) .
\end{aligned}
$$

Thus $G(w, w, h w)+G(z, z, h z)=0$. So, we get

$$
h w=R(w, z)=w \quad \text { and } \quad h z=R(z, w)=z .
$$

Hence from (3), (4) and (5) we conclude that $(w, z)$ is the same common coupled fixed point of the pairs $(f, S),(g, T)$ and $(h, R)$.

Now, we prove the uniqueness. assume that $\left(w^{*}, z^{*}\right)$ is another same common coupled fixed point of the pairs $(f, S),(g, T)$ and $(h, R)$. By (1) we get

$$
\begin{aligned}
& G\left(S(w, z), T(w, z), R\left(w^{*}, z^{*}\right)\right)+G\left(S(z, w), T(z, w), R\left(z^{*}, w^{*}\right)\right) \\
& \leq k\left(G\left(f w, g w, h w^{*}\right)+G\left(f z, g z, h z^{*}\right)\right),
\end{aligned}
$$

which yields that

$G\left(w, w, w^{*}\right)+G\left(z, z, z^{*}\right) \leq k\left(G\left(w, w, w^{*}\right)+G\left(z, z, z^{*}\right)\right)$, Hence $w=w^{*}$ and $z=z^{*}$.

Now, we show that $w=z$. From (1) ones obtain

$$
\begin{aligned}
& G(S(w, z), T(w, z), R(z, w))+G(S(z, w), T(z, w), R(w, z)) \\
& \leq k(G(f w, g w, h z)+G(f z, g z, h w)) .
\end{aligned}
$$

Then

$$
G(w, w, z)+G(z, z, w) \leq k(G(w, w, z)+G(z, z, w)) .
$$

Hence $w=z$. Therefore $(w, w)$ is the same unique common coupled fixed point of the pairs $(f, S),(g, T)$ and $(h, R)$. The proof is similar if $g(X)$ or $h(X)$ is a $G$-complete subspace of $X$.

If we put $f=g=h=I$ (where $I$ is the identity mapping) we have the following corollary.

Corollary 1.Let $(X, G)$ be a complete $G$-metric space. Let $S, T, R: X \times X \rightarrow X$ be mappings satisfying the following condition

$$
\begin{aligned}
& G(S(x, y), T(u, v), R(w, z))+G(S(y, x), T(v, u), R(z, w)) \\
& \leq k(G(x, u, w)+G(y, v, z)),
\end{aligned}
$$

for all $x, y, u, v, w, z \in X$, where $k \in[0,1)$. Then $S, T$ and $R$ have a a unique common coupled of the form $(w, w)$ in $X \times X$. 
If we put $S=T=R$ and $f=g=h$ we have the following corollary.

Corollary 2.Let $(X, G)$ be a $G$-metric space. Let $S: X \times$ $X \rightarrow X$ and $f: X \rightarrow X$ be mappings satisfying the following condition

$$
\begin{aligned}
& G(S(x, y), S(u, v), S(w, z))+G(S(y, x), S(v, u), S(z, w)) \\
& \leq k(G(f x, f u, f w)+G(f y, f v, f z)),
\end{aligned}
$$

for all $x, y, u, v, w, z \in X$, where $k \in[0,1)$. Suppose that

(i) $S(X \times X) \subseteq f(X)$,

(ii) $f(X)$ is a $G$-complete subspace of $X$.

(iii)the pair $(f, S)$ is w-compatible.

Then $f$ and $S$ have a unique common coupled fixed point of the form $(w, w)$ in $X \times X$.

Now, we give an example to justify the hypotheses of Theorem 1.

Example 1.Let $X=[0,1]$. Define $G: X \times X \times X \rightarrow X$ by

$$
G(x, y, z)=\max \{|x-y|,|y-z|,|z-x|\} .
$$

Let S, $T, R: X \times X \rightarrow X$ and $f, g, h: X \rightarrow X$ be defined by

$$
\begin{gathered}
S(x, y)=\frac{x+3 y}{12}, \quad T(x, y)=\frac{x+3 y}{16}, \quad R(x, y)=\frac{x+3 y}{14} \\
f x=\frac{2 x}{3}, \quad g x=\frac{x}{2}, \quad h x=\frac{4 x}{7} .
\end{gathered}
$$

We see that $S(X \times X)=\left[0, \frac{1}{3}\right] \subseteq g(X)=\left[0, \frac{1}{2}\right], T(X \times X)=$ $\left[0, \frac{1}{4}\right] \subseteq h(X)=\left[0, \frac{4}{7}\right], R(X \times X)=\left[0, \frac{2}{7}\right] \subseteq f(X)=\left[0, \frac{2}{3}\right]$. Also, the pairs $(f, S),(g, T)$ and $(h, R)$ are $w$-compatible.

Now, for all $x, y, u, v, w, z \in X$ we obtain

$$
\begin{aligned}
& G(S(x, y), T(u, v), R(w, z))+G(S(y, x), T(v, u), R(z, w)) \\
& =\max \left\{\left|\frac{x+3 y}{12}-\frac{u+3 v}{16}\right|,\left|\frac{u+3 v}{16}-\frac{w+3 z}{14}\right|,\left|\frac{w+3 z}{14}-\frac{x+3 y}{12}\right|\right\} \\
& +\max \left\{\left|\frac{y+3 x}{12}-\frac{v+3 u}{16}\right|,\left|\frac{v+3 u}{16}-\frac{z+3 w}{14}\right|,\left|\frac{z+3 w}{14}-\frac{y+3 x}{12}\right|\right\} \\
& \leq \max \left\{\left|\frac{x}{12}-\frac{u}{16}\right|,\left|\frac{u}{16}-\frac{w}{14}\right|,\left|\frac{w}{14}-\frac{x}{12}\right|\right\}+\max \left\{\left|\frac{3 y}{12}-\frac{3 v}{16}\right|,\left|\frac{3 v}{16}-\frac{3 z}{14}\right|,\left|\frac{3 z}{14}-\frac{3 y}{12}\right|\right\} \\
& +\max \left\{\left|\frac{y}{12}-\frac{v}{16}\right|,\left|\frac{v}{16}-\frac{z}{14}\right|,\left|\frac{z}{14}-\frac{y}{12}\right|\right\}+\max \left\{\left|\frac{3 x}{12}-\frac{3 u}{16}\right|,\left|\frac{3 u}{16}-\frac{3 w}{14}\right|,\left|\frac{3 w}{14}-\frac{3 x}{12}\right|\right\} \\
& =4 \max \left\{\left|\frac{x}{12}-\frac{u}{16}\right|,\left|\frac{u}{16}-\frac{w}{14}\right|,\left|\frac{w}{14}-\frac{x}{12}\right|\right\}+4 \max \left\{\left|\frac{y}{12}-\frac{v}{16}\right|,\left|\frac{v}{16}-\frac{z}{14}\right|,\left|\frac{z}{14}-\frac{y}{12}\right|\right\} \\
& =\frac{1}{2}\left(\max \left\{\left|\frac{2 x}{3}-\frac{u}{2}\right|,\left|\frac{u}{2}-\frac{4 w}{7}\right|,\left|\frac{4 w}{7}-\frac{2 x}{3}\right|\right\}+\max \left\{\left|\frac{2 y}{3}-\frac{v}{2}\right|,\left|\frac{v}{2}-\frac{4 z}{7}\right|,\left|\frac{4 z}{7}-\frac{2 y}{3}\right|\right\}\right) \\
& \leq \frac{3}{4}(G(f x, g u, h w)+G(f y, g v, h z)) .
\end{aligned}
$$

The hypotheses of Theorem 1 are holds with constant $k=$ $\frac{3}{4}$. Also, $(0,0)$ is the same unique common coupled fixed point of the pairs $(f, S),(g, T)$ and $(h, R)$.

\section{References}

[1] H. Aydi, B. Damjanović, B. Samet, and W. Shatanawi, Coupled fixed point theorems for nonlinear contractions in partially ordered G-metric spaces, Mathematical and Computer Modelling, 54, 2443-2450 (2011).
[2] M. Abbas, M. A. khan, S. Radenovic, Common coupled fixed point theorems in cone metric spaces for $w$-compatible mappings, Applied Mathematics and Computation, 217, 195202 (2010).

[3] T. G. Bhaskar, V. Lakshmikantham, Fixed point theorems in partially ordered metric spaces and applications, Nonlinear Anal., 65, 1379-1393 (2006).

[4] B.S. Choudhury, P. Maity, Coupled fixed point results in generalized metric spaces, Math. Comput. Modelling, 54, 7379 (2011).

[5] H-S. Ding, E. Karapnar, A note on some coupled fixed-point theorems on $G$-metric spaces, Journal of Inequalities and Appl., 2012, 170 (2012).

[6] D. Guo, V. Lakshmikantham, Coupled fixed points of nonlinear operators with applications, Nonlinear Anal., 11, 623-632 (1987).

[7] Erdal Karapinar, Coupled fixed point theorems for nonlinear contractions in cone metric spaces, Comput. Math. Appl., 59, 3656-3668 (2010).

[8] V. Lakshmikantham, Lj. B.Ciric, Coupled fixed point theorems for nonlinear contractions in partially ordered metric spaces, Nonlinear Anal., 70, 4341-4349 (2009).

[9] N. V. Luong and N. X. Thuan, Coupled fixed point theorems in partially ordered metric spaces, Bull Math Anal Appl., 2, 16-24 (2010).

[10] Z. Mustafa and B. Sims, Some remarks concerning $D$-metric spaces, Intern. Conf. Fixed Point. Theory and Applications, Yokohama, 189-198 (2004).

[11] Z. Mustafa and B. Sims, A new approach to generalized metric spaces, J. Nonlinear Convex Analysis, 7, 289-297 (2006).

[12] Z. Mustafa and B. Sims, Fixed point theorems for contractive mappings in complete $G$-metric spaces, Fixed Point Theory and Applications, Article ID 917175, 2009, 10 pages (2009).

[13] W. Shatanawi, Partially ordered cone metric spaces and coupled fixed point results, Computers Math. Appl., 60, 2508-2515 (2010).

[14] W. Shatanawi, B. Samet and M. Abbas, Coupled fixed point theorems for mixed monotone mappings in ordered partial metric spaces, Math. Comput. Modelling, (2011).

[15] B. Samet, Coupled fixed point theorems for a generalized Meir-Keeler contraction in partially ordered metric spaces, Nonlinear Anal., 72, 4508-4517 (2010).

[16] B. Samet, H. Yazidi, Coupled fixed point theorems in partially ordered -chainable metric spaces, TJMCS., 1, 142151 (2010). 